\title{
Vacuole-specific lipid release for tracking intracellular lipid metabo- lism and transport in Saccharomyces cerevisiae
}

\author{
Vladimir Girik‡, Suihan Feng‡, Hanaa Hariri, W Mike Henne, Howard Riezman*
}

\begin{abstract}
Lipid metabolism is spatiotemporally regulated within cells, yet intervention into lipid functions at subcellular resolution remains difficult. Here we report a method that enables to site-specifically release sphingolipids and cholesterol inside the vacuole in Saccharomyces cerevisiae. Using this approach, we monitored real-time sphingolipid metabolic flux out of vacuole by mass spectrometry and found that the ER-vacuole tethering protein Mdm1 facilitated the metabolism of sphingoid bases into ceramides. In addition, we showed that cholesterol, once delivered into yeast using our method, could restore cell proliferation induced by ergosterol deprivation, overcoming the previously described sterol-uptake barrier under aerobic conditions. Together, these data define a new way to modulate sterol composition in yeast and study intracellular lipid metabolism and transport from the vacuole in yeast.
\end{abstract}

\section{INTRODUCTION}

Lipids are heterogeneously distributed in eukaryotic cells with their composition largely varying among the specific subcellular compartments. ${ }^{1}$ This heterogeneity is achieved despite the fact that most lipid species are first synthesized in one organelle and transported to other organelles for use or further modification. Although most enzymes responsible for lipid metabolism have been identified, how lipids are transported within cells is much less known and many lipid transport routes are still waiting to be established.

In recent years, a number of lipid transfer protein (LTP) have been found to facilitate non-vesicular lipid transport through contact sites between organelles. ${ }^{2}$ In many cases, structural information and in vitro lipid transfer assays need to be established in order to validate the roles of LTPs in lipid transport. This can be a challenging task because many LTPs are transmembrane proteins and lack detailed structural information. Mass spectrometry (MS)-based lipidomics techniques can contribute to our understanding of transport by providing quantitative analysis over a large number of chemically diverse lipid species. Designed to carry out lipidome-wide scans, it has been shown particularly useful when combined with genetic perturbation in yeast cells. ${ }^{3-5}$ Nevertheless, the results of gene deletion cannot always translate into lipid profile changes. For example, simply removing one LTP may not generate changes in lipid profiles if a lipid transport route is maintained by several proteins with overlapping functions, as is often the case. Clearly, it is desirable to develop new approaches which overcome functional redundancy issues and do not rely on in vitro protein-lipid reconstruction.

Previously, we have developed a series of coumarin-based photo-cleavable (caged) lipid probes targeting to mitochondria and lysosomes, respectively. ${ }^{6,} 7$ Upon illumination, these probes quickly decomposed, thereby releasing the correspond- ing native lipid molecules inside the targeted organelle. Using isotope-labelled sphingosine as a lipid precursor, we detected its metabolic products by mass spectrometry with high sensitivity, and showed that sphingolipid metabolic patterns highly depend on subcellular localization. ${ }^{6}$ While this strategy is firmly established in mammalian cells, it was unclear whether the same concept is applicable to yeast, an extensively used model organism for studying lipid homeostasis owing to its simplicity, ease of genetic manipulation and similar organization to metazoans.

To explore their use in yeast, we applied mitochondrialtargeted caged probes onto Saccharomyces cerevisiae (S. cerevisiae), and found surprisingly that these probes accumulated inside the vacuole instead of mitochondria. This unexpected finding led us to synthesize a series of caged lipids, including phytosphingosine (PHS) and cholesterol, to identify regulators of the vacuole specific accumulation (Figure 1). Next, we used TPPCou-C17PHS as a model compound to characterize the metabolic products of vacuolar sphingoid bases. Using mass spectrometry as a readout, we investigated potential effectors en route to the sphingolipid metabolic pathway, and provided clear evidence that an ER anchored protein which also contacts the vacuole surface, Mdm1, facilitated conversion of vacuolar sphingoid base into dihydroceramide. Additionally, we successfully delivered cholesterol into $S$. cerevisiae using the same strategy. We showed that cholesterol can restore cell proliferation induced by ergosterol deprivation, and that deletion of NCR1 did not block vacuolederived cholesterol utilization in yeast cells, unlike its mammalian homolog NPC1, which is thought to be important for cholesterol transport in mammals., 9 Collectively, our approach, which combines synthesis, imaging, lipidomics and genetic modification, demonstrated a new way of studying intracellular lipid transport and metabolism in S. cerevisiae. 

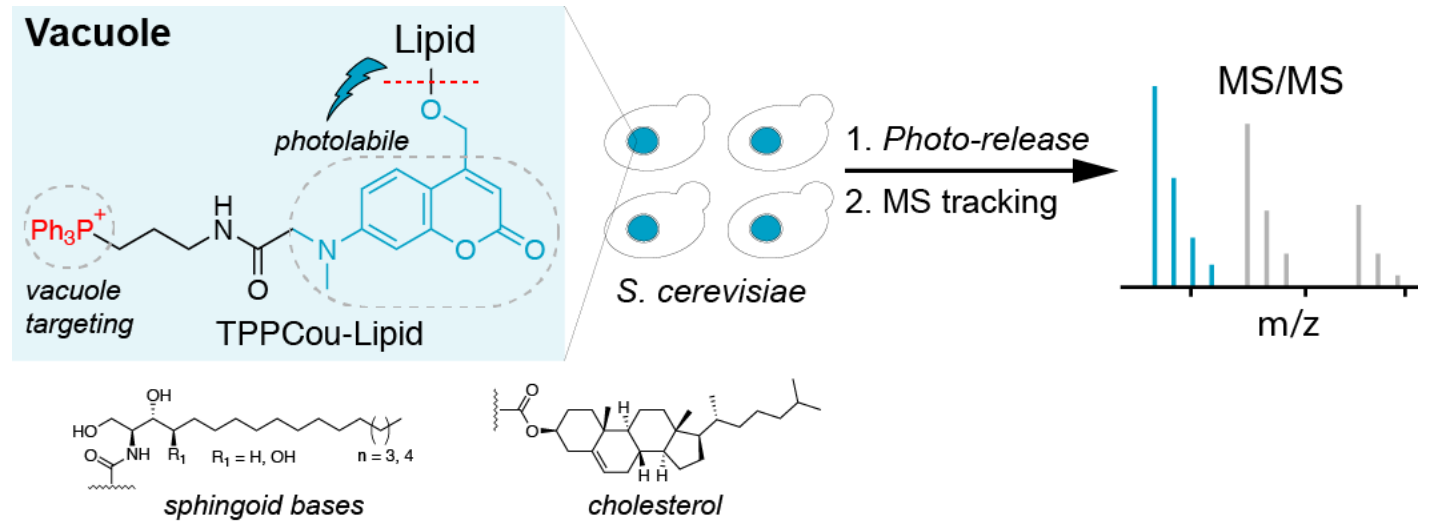

Figure 1. Schematic illustration of MS-based metabolic tracking of vacuole-targeted photocleavable (TPPCou) lipid probes.

\section{RESULTS}

TPPCou-caged sphingoid bases accumulated in yeast vacuole. Triphenylphosphonium (TPP) cation is a well-known mitochondrial targeting motif and has been used to deliver a wide range of small molecules into mitochondrial matrix in mammalian cells ${ }^{10}$. In the past, we have used TPP-modified coumarin as a photo-cleavable protecting group to synthesize caged sphingolipids targeting to the mitochondria in mammalian cells. The localization was clearly visualized under fluorescence microscopy, because the coumarin that we used is also a fluorophore apart from being a photo-cleavable protecting group. Since it was unclear whether these caged probes accumulate in yeast mitochondria too, we incubated the caged sphinganine (Mito-Sa) in S. cerevisiae together with a mitochondrial marker, Mdh1-mCherry. To our surprise, while we recorded a strong fluorescence signal in the coumarin channel, the staining pattern did not overlap the Mdh1-mCherry signal, but rather looked similar to the vacuole. This unexpected finding prompted us to examine co-localization with a vacuole
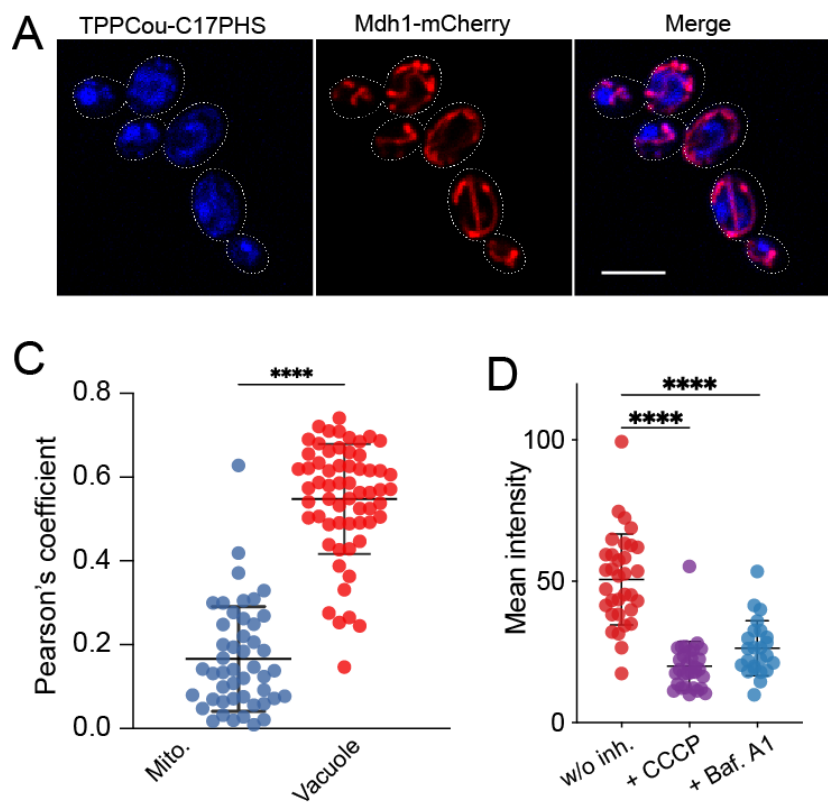

marker, and we consistently found that indeed the coumarin signal overlapped well with the vacuole marker, Vph1mCherry (Supplementary Figure 1). Owing to the results that Mito-Sa localized to the mitochondria in mammalian cells and to the vacuole in yeast cells, we renamed it as TPPCou-C18Sa in order not to cause any further confusion.

Phytosphingosine (PHS) is the major form of long chain sphingoid bases (LCBs) in yeast ${ }^{11}$, so TPPCou-C17PHS was eventually chosen in our study in order to differentiate it in mass spectrometry from the endogenous PHS which predominantly has an 18-carbon chain. Accordingly, we prepared the TPPCou-C17PHS and other caged lipids following established procedures which have been described in our previous studies (Figure 1) ${ }^{6,7}$ Using fluorescence microscopy, we have found, consistently, that TPPCou-C17PHS accumulates inside the vacuole, but not the mitochondria (Figure $2 \mathrm{~A}, \mathrm{~B}, \mathrm{C}$ ). To learn which factors affect the vacuole staining, we treated the cells with diethylaminocoumarin-caged PHS (Cou-C18PHS) without the TPP cation and TPPCou-OH alone, respectively (Supplementary Figure 2 B-C), but failed to observe any meaning-
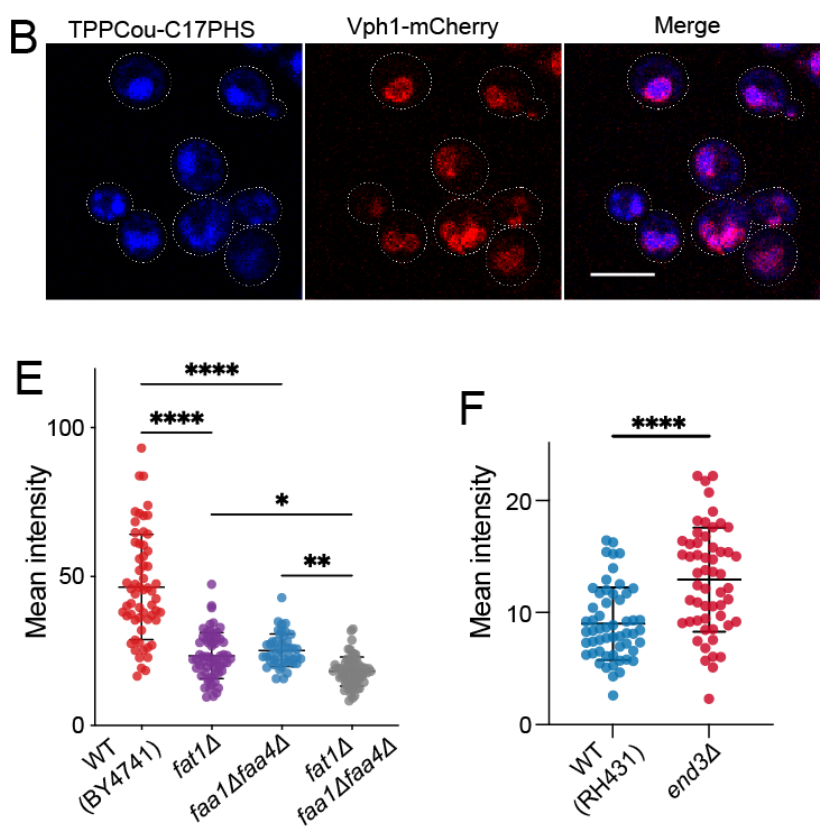

Figure 2. TPPCou-C17PHS accumulated in the yeast vacuole. (A) and (B) Representative confocal images of yeast cells stably expressing mCherry-tagged (red) mitochondrial protein Mdh1 (A) or vacuolar protein Vph1 (B) stained with $10 \mu \mathrm{M}$ TPPCou-C17PHS (blue). Bars, $5 \mu$ m (C) Quantification of colocalization of TPPCou-C17PHS with mCherry-Mdh1 and mCherry-Vph1, respectively. (D, E, F) Quantification of mean intracellular fluorescence intensity of TPPCou-C17PHS. Cells were treated with $20 \mu \mathrm{M}$ TPPCou-C17PHS for 15 min in the presence of indicated conditions. ${ }^{*} \mathrm{p}<0.05,{ }^{* *} \mathrm{p}<0.01, * * * * \mathrm{p}<0.0001$, ns., not significant, student's t-test. 
ful fluorescence signals, indicating that both the TPP cation and a lipid chain are compulsory for effective transport of caged probes into the vacuole. Next, we treated the cells with TPPCou-C17PHS in the presence of CCCP and bafilomycin A1, respectively. Either disrupting the proton gradient with CCCP or blocking vacuolar V-ATPases with bafilomycin significantly reduced the fluorescence intensities of TPPCouC17PHS (Figure 2D), suggesting that the vacuole staining is dependent on the $\mathrm{pH}$ gradient across the vacuolar membrane. We postulated that proteins involved in long-chain base uptake may play a role in the internalization of TPPCou-C17PHS. Indeed, we found a significant decrease of fluorescence signals in the absence of fatty acyl-CoA synthetases (faa1, faa4) and/or transporter (fat1), which have been shown to affect uptake of exogenous sphingoid bases ${ }^{12}$ (Figure 2E). We furthermore investigated whether the uptake of TPPCou-C17PHS was via endocytosis. Using cells that lack END3, essential for endocytosis, ${ }^{13}$ we found that the fluorescence intensities from end3 cells are even slightly higher than the ones from the wild type (WT) cells (Figure 2F), suggesting that the transport does not rely on endocytosis. Notably, the average fluorescence intensities in both conditions (Figure 2F) are relatively lower compared to other experiments (Figure 2D, E), likely caused by the differences among the yeast strain backgrounds. To summarize, we showed that the TPPCou-caged lipids accumulated inside the yeast vacuole, and that elements crucial for the import and localization include the proton gradient across the vacuolar membrane and proteins required for sphingoid base and fatty acid import.

Metabolism of vacuole-released C17PHS. The lyso-

A
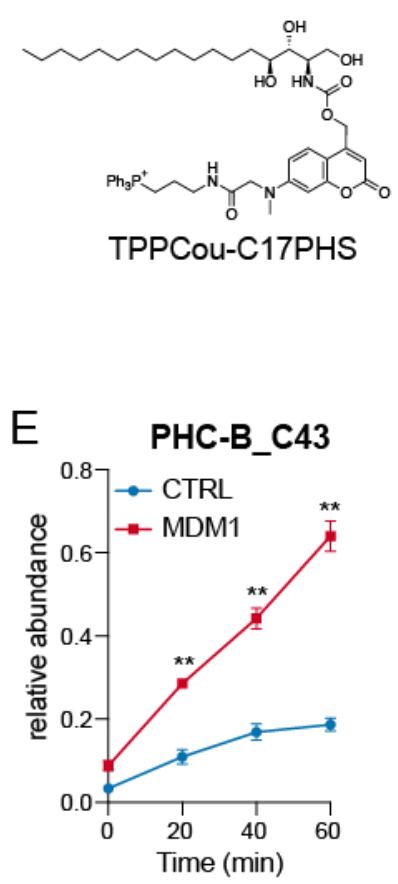

$\mathrm{B}$
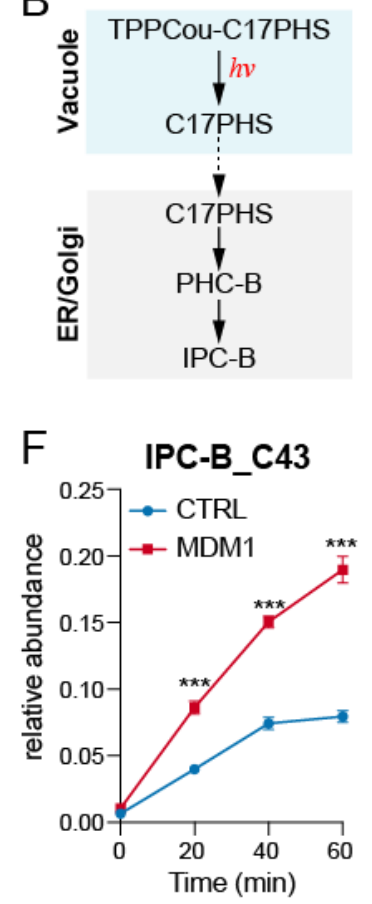

some/vacuole serves as a primary site to break down complex lipid molecules into basic build blocks, which are later transported to other sites such as endoplasmic reticulum (ER) for lipid synthesis ${ }^{14}$. These processes of storage and recycling are of fundamental importance and have been linked to a number of diseases, but many aspects, particularly on the sphingolipid transport, remain elusive ${ }^{15}$. Previously, we have applied local uncaging methods in mammalian cells to show that sphingolipid metabolism and turnover have distinct properties depending on the subcellular localization of the release ${ }^{6}$, though lipid transport was not defined and remains elusive. Here we applied the same principle to examine vacuole-released sphingolipid metabolism, with a particular focus on lipid transport.

The vacuole maintains a low $\mathrm{pH}$ environment and hosts a range of enzymes to disassemble lipid molecules. To assess the stability of caged probes after import into the vacuole, cells were treated with TPPCou-C17PHS, collected before or after UV illumination, and analyzed by mass spectrometry (Figure 3C). We detected C17PHS even without illumination, likely due to undesired enzymatic activities in the vacuole. Nevertheless, the majority remained intact, judging from the UV light-released C17PHS, which is about four times higher than the control. After learning that the majority of the caged probe was intact, we analyzed its metabolic products after uncaging and compared to the ones delivered by extracellular addition of C17PHS. Phytosphingosine can be transported from the vacuole to the nuclear ER where it is converted into various ceramides and other complex sphingolipids (Figure 3B). Our analysis showed that exogenously added C17PHS was predominantly converted into phytoceramide_C43:0

C
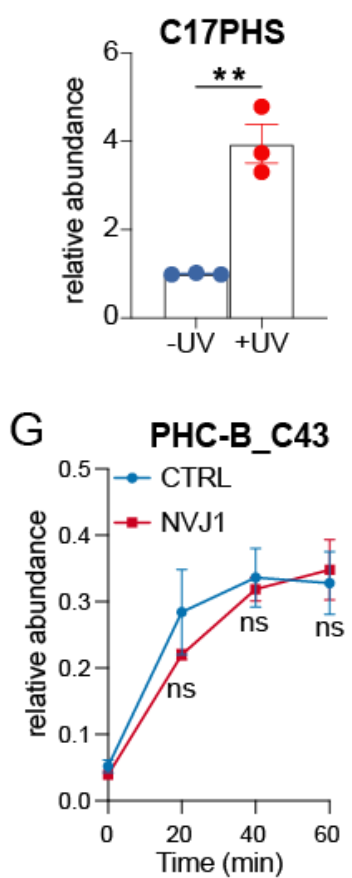
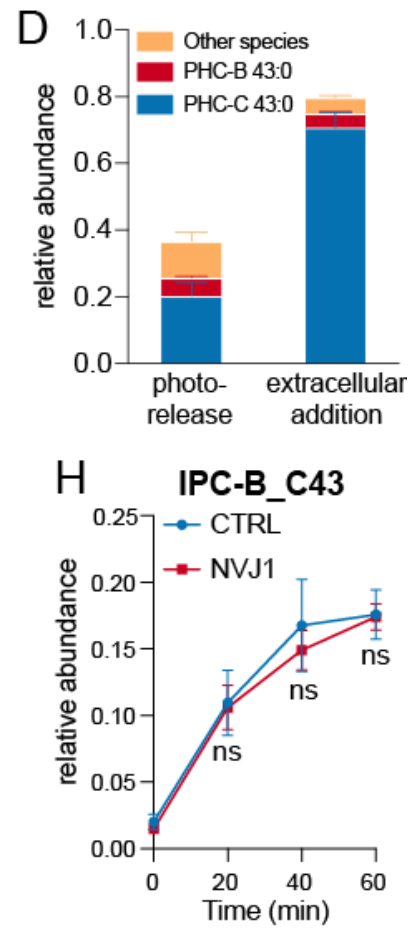

Figure 3. Metabolism of vacuole-released C17PHS. (A) Chemical structure of TPPCou-C17PHS (B) Metabolic scheme of C17PHS after the vacuole release. After transport from the vacuole to the ER, PHS is converted into Phytoceramide (PHC-B) which is transported further to the Golgi, where PHC-B is metabolized into Inositol Phosphorylceramide (IPC-B). (C) Analysis of C17PHS levels before (-UV) and after(+UV) UV uncaging in cells labeled with $5 \mu \mathrm{M}$ TPPCou-C17PHS. D) Profile of ceramide species derived from TPPCou-C17PHS (after photo-release) or extracellularly added C17PHS. (E-H) Lipid analysis of sphingolipids derived from vacuole-released C17PHS after UV uncaging in cells overexpressing empty vector, MDM1 (E-F) or NVJ1 (G-H). Data represents the average of three independent experiments. Error bars represent SEM. ** $<0.01$, $* * * \mathrm{p}<0.001$, ns, not significant, students' t-test. 
bioRxiv preprint doi: https://doi.org/10.1101/2021.05.04.442581; this version posted May 4, 2021. The copyright holder for this preprint (which was not certified by peer review) is the author/funder, who has granted bioRxiv a license to display the preprint in perpetuity. It is made available under aCC-BY-NC-ND 4.0 International license.

(PHC_C43:0). In photo-released cells, however, a significantly higher proportion of C17PHS was used for the synthesis of PHC_B and other ceramide species (Figure 3D). Although the amount of C17PHS being delivered into intracellular space was different under the two experimental conditions, these results clearly revealed subcellular dependence of sphingolipid metabolism, consistent with our previous findings in mammalian cells. ${ }^{6}$

In $S$. cerevisiae, the levels of long chain sphingoid bases (LCB) are tightly regulated by coordinated action of metabolic enzymes ${ }^{16}$. We have previously reported that LCB kinases are essential for incorporating exogenous LCBs into complex sphingolipids ${ }^{17}$. It is therefore interesting to know whether the vacuole-released sphingolipids are also subjected to regulation of LCB kinases. To this end, we prepared deuterated TPPCouSa_D7 and analyzed its metabolic products after photoreleasing in wide type (WT) and mutant cells lacking LCB4 ( $\Delta l c b 4)$, respectively (Supplementary Figure 3). In parallel, we used exogenously added Sa_D7 as a control and observed that the converted sphingolipids such as PHC and IPC were drastically reduced in $\Delta l c b 4$ cells (Supplementary Figure 3C, D). Decreased sphingolipid conversion was also found in the vacuole-released Sa_D7 experiments, but to a much lesser extent (Supplementary Figure 3E, F). These results indicate that metabolism of vacuolar LCBs is much less dependent on the LCB kinases, underscoring the complexity of lipid homeostasis and the importance of paying attention to local lipid metabolic routes.

MDM1 facilitated sphingolipid turnover. Lipids can be transported within cells by two pathways, vesicular and nonvesicular, but for most lipids it is thought that the majority of traffic is carried out by non-vesicular trafficking, most likely involving lipid transfer proteins and membrane contact sites (MCS), areas where membranes from two organelles are in close apposition ${ }^{18}$. A number of imaging-based studies indi-

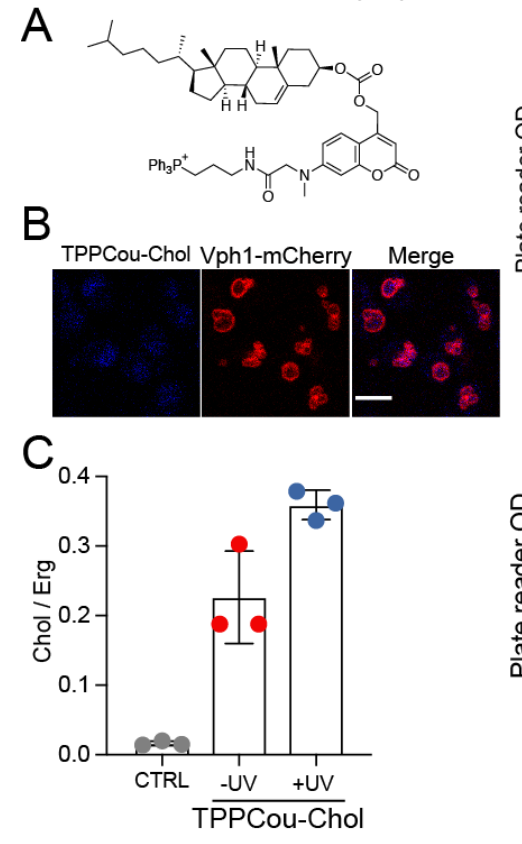

cated the universal presence of MCS, but functional studies are still lagging behind ${ }^{19}$. In the recent years, we have identified MDM1 as a tethering protein which localized to the ER but forms contacts with vacuole and lipid droplets in yeast. ${ }^{20,21}$ MDM1 is highly conserved in metazoans and plays essential roles in regulating lipid homeostasis ${ }^{22-24}$. We have found that mutations in yeast MDM1 perturb sphingolipid metabolism, but whether this tether is involved in inter-organelle lipid trafficking remained elusive ${ }^{25}$.

Seeking to elucidate potential functions of MDM1 in ERvacuole crosstalk, we used the vacuole-specific uncaging concept to examine whether sphingolipid turnover is influenced by MDM1 over time. Accordingly, we developed a pipeline in which C17PHS was first released inside the vacuole, and its metabolic products were measured by mass spectrometry at different time points. PHC-B_C43 and IPC-B_C43 are two of the major metabolic products derived from $\mathrm{C} 17 \mathrm{PHS}$ and gave lowest signal-to-noise ratios, and hence were selected in our analysis. Our data showed that the accumulation of both PHCB_C43 and IPC-B_C43 were significantly higher in cells in which MDM1 was overexpressed (Figure 3E, F). However, when we overexpressed $N V J 1$, another established nucleusvacuole junction (NVJ) tether, ${ }^{26}$ we did not observe any meaningful difference when compared to the control cells (Figure 3G, H). This striking contrast between MDM1 and NVJ1, which both promote increased MCS formation, indicates that MDM1 facilitated the turnover of vacuolar sphingolipids by additional means than its role in MCS formation.

Intriguingly, when we attempted to use MDM1 or NVJ1 knockout strains in the 'pulse-chase' experiments, we detected high levels and variable quantities of endogenous C17 sphingolipids. Since those sphingolipids share the same chemical structures with the ones converted from the C17PHS after uncaging, it was difficult to deduce any meaningful conclusion from those experiments. The reason for the appearance of odd-
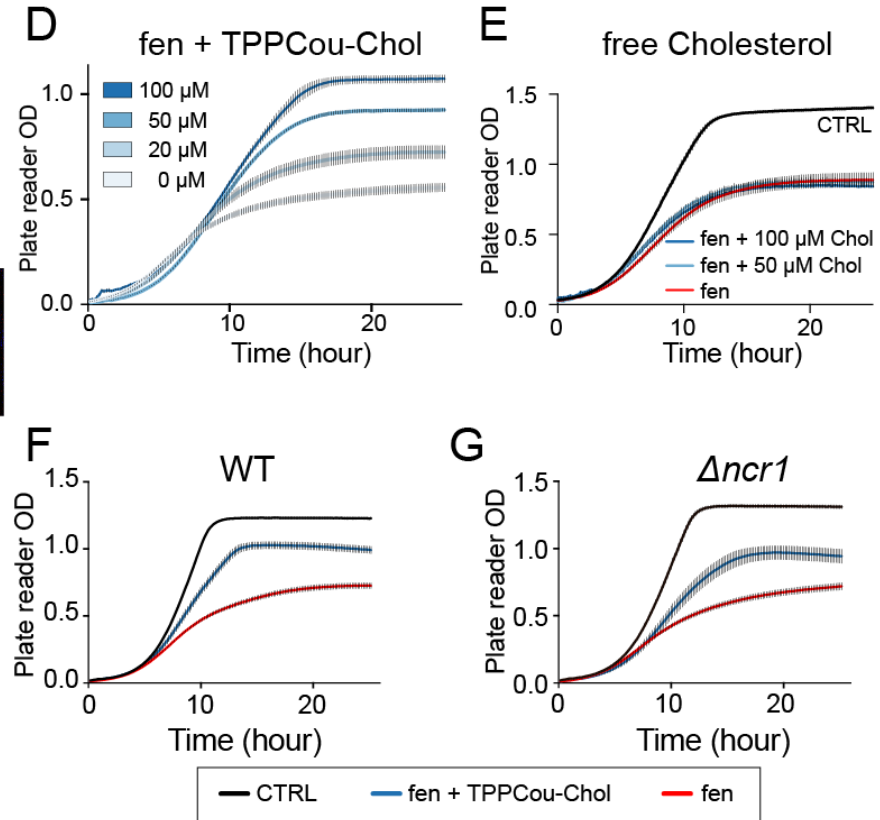

Figure 4. Vacuole-released cholesterol rescues growth of ergosterol-depleted yeast cells. (A) Chemical structure of TPPCou-Chol. (B) Representative confocal images of yeast cells stably expressing mCherry-tagged (red) vacuolar protein Vph1 stained with $50 \mu \mathrm{M}$ TPPCou-Chol (blue) for $20 \mathrm{~min}$ at $30^{\circ} \mathrm{C}$. Bars, $5 \mu \mathrm{m}$. (C) GC-MS analysis of vacuole-released cholesterol in yeast cells. Cholesterol amounts are shown as percentage of total ergosterol levels. Data represents the average of three biological replicates. Error bars represent SD. (D) Growth curves of cells treated with different amount of TPPCou-Chol as indicated in the presence of fenpropimorph $(8 \mu \mathrm{M})$. (E) Growth curves of cells treated with free cholesterol in the presence of fenpropimorph $(8 \mu \mathrm{M})$, or ethanol (CTRL) as indicated. (F, G) Growth curves of WT or ncr1 $\Delta$ mutant cells. Cells were grown in presence of fenpropimorph $(8 \mu \mathrm{M})$ and TPPCou-Chol. Cells were incubated in a plate reader and the growth was recorded by taking regular OD measurements at $30^{\circ} \mathrm{C}$. Individual values represent the average of four biological replicates. Error bars represent SEM. 
chain sphingolipids in these mutants is unknown. Since neither MDM1 nor NVJ1 is essential for cell growth, cells do not seem to rely on a single component/pathway to transport lipids between the vacuole and the ER. The functional redundancy and/or quick metabolic adaptation could also mean that overexpression rather than gene deletion is more suitable to capture differences in the metabolic tracking studies using our method. In addition, our lipidomics analysis indicated that major lipid profiles were not significantly shifted after the photo-release (Supplementary Figure 5), likely because the LCBs delivered to the cells only comprise a very small portion of the endogenous lipid pool.

Cholesterol delivered to yeast using TPPCou as carrier. Cholesterol is a fundamentally important molecule to human health, yet its physiological roles are still not clearly defined and some are highly debated ${ }^{27}$. This is partially because cholesterol is so abundant and essential in mammalian cells that any experimental means for reducing cholesterol levels faces the risk of jeopardizing numerous undesired cellular functions. In contrast, wild type yeast cells do not produce cholesterol and use another sterol, ergosterol, for maintaining cellular activities. Having engineered a yeast strain that effectively produces cholesterol, we demonstrated that functions of ergosterol can be partially replaced by cholesterol, which makes yeast an attractive model organism for studying cholesterol transport and metabolism ${ }^{28}$. However, while mammalian cells import cholesterol through receptor mediated endocytosis, $S$. cerevisiae does not take up sterols under aerobic conditions ${ }^{29}$.

To gain insights into cholesterol transport, we prepared TPPCou-caged cholesterol (TPPCou-Chol, Figure 4A) and inspected whether it can be effectively delivered to the vacuole in wild type yeast cells. As expected, the probe was successfully delivered into the vacuole (Figure 4B), similar to the TPPCou-C17PHS. In addition, we measured the cholesterol uptake under various conditions and found that cholesterol was indeed delivered to the cells using TPPCou-Chol, although there was only minimal additional effect due to UV illumination (Figure 4C). These results suggest that most TPPCou-Chol was cleaved by vacuolar enzymes prior to UV exposure, which also partially explains why the fluorescence signals from TPPCou-Chol were weaker than the ones from TPPCou-caged LCBs (Figure 4B). Despite lacking optical control on the TPPCou-Chol, the data marked the successful vacuole-targeted delivery of cholesterol under aerobic conditions, enabling us to further investigate cholesterol transport in S. cerevisiae. To this end, we blocked endogenous ergosterol biosynthesis using fenpropimorph ${ }^{30}$, and treated cells with TPPCou-Chol in increasing amounts, and monitored the cell growth. The growth curves indicated that TPPCou-Chol effectively rescued the deficiency of ergosterol in a dose-dependent manner (Figure 4D), while free cholesterol treated cells failed to alleviate any cell growth suppressed by fenpropimorph (Figure 4E). Using fenpropimorph treated cells, we also performed another set of experiments on NCR1 knockout cells. We observed similar growth curves positively correlated with the amount of TPPCou-Chol, but did not see any significant difference between wild type (WT) and NCR1 knock cells (Ancr1) (Figure 4F, 4G). Our results suggest that NCR1, unlike its mammalian homolog NPC $1,{ }^{8}$ is less important for the flux of sterols from the lysosomes/vacuoles, in agreement with pervious findings. ${ }^{9}$ Overall, our data showed that the vacuolespecific uncaging approach is not limited to sphingoid bases and has the potential for broader application. Furthermore, it has established a novel protocol to introduce sterols into aerobically grown yeast which should permit future studies on the function of sterols in cell biology.

\section{DISCUSSION}

In eukaryotic cells, distinct lipid distribution and metabolism are associated with subcellular compartments, but related tools to directly address these issues are very limited. Recently, we have developed a local uncaging strategy that offers direct experimental means to study organelle-specific lipid metabolism. Here, we introduced this concept to the yeast system and extended its application to intracellular lipid transport. Together with mass spectrometry, we provide quantitative information of sphingolipid metabolism and unveiled the active role of MDM1 in facilitating the conversion of vacuolar long chain sphingoid bases (LCBs) into ceramides. In addition, by releasing cholesterol in the vacuole, we showed that cholesterol can effectively rescue the deficiency of ergosterol, that cholesterol utilization was not hindered by the removal of NCR1 and that it is possible using this method to introduce sterols into aerobically grown yeast.

The fluorescence imaging data shows that TPPCou-caged lipids accumulated in the vacuole, but it is still unclear why they are enriched inside the vacuole instead of mitochondria. Triphenylphosphonium (TPP) cation is known as a mitochondrial targeting signal and has been extensively used in mammalian systems, ${ }^{10}$ yet we did not find any targeting to mitochondria in yeast. Although it is difficult to rule out the possibility that some of the TPPCou-based probes went into mitochondria, the amount accumulated in mitochondria is under the detection threshold and thus must be limited. We explored the uptake mechanisms of TPPCou-caged lipids using knockout strains, and our results show that probe uptake involves the participation of acyl-CoA synthases but independent of endocytosis. In addition, experiments using CCCP and bafilomycin indicate that the proton gradient is essential to localize the probes inside the vacuoles. Since the vacuole is a recycling hub for breaking down complex lipids into building blocks, it is not a surprise that we observed partial hydrolysis of TPPCou-C17PHS prior to UV illumination, and that TPPCou-Chol was completely decomposed. On the other hand, the successful delivery of long chain sphingoid bases and cholesterol also suggests a possible broader application of using TPPCou as cargo to transport other lipid molecules to the vacuole in $S$. cerevisiae.

The major lipid trafficking routes are organized by nonvesicular lipid transport, particularly through membrane contact sites (MCS) with the involvement of a large group of lipid transfer proteins (LTP). A number of imaging-based studies revealed the precise localization of these proteins, but functional studies often lag behind. We released phytosphingosine in the vacuole and monitored its metabolic products over time, in an attempt to establish a system which can directly measure the influence of LTPs on lipid movement and metabolism. Our results showed that overexpressing MDM1, an ER-vacuole tethering protein, facilitated formation of ceramide and IPC species, unlike another junction protein, NVJ1. Since ceramide synthases are localized to the nuclear $E R,{ }^{31}$ active lipid transport from vacuole to the ER should be prerequisite before the metabolic conversion. We have previously shown that MDM1 mutants suppress cell survival in myriocin treated plating assays ${ }^{25}$, but details were lacking because suppressing 
bioRxiv preprint doi: https://doi.org/10.1101/2021.05.04.442581; this version posted May 4, 2021. The copyright holder for this preprint (which was not certified by peer review) is the author/funder, who has granted bioRxiv a license to display the preprint in perpetuity. It is made available under aCC-BY-NC-ND 4.0 International license.

sphingolipid biosynthesis by myriocin can have profound effects on numerous aspects of cellular activities. In our current study, we measured the real-time metabolic flux of sphingolipids originated from vacuole, thus provided a direct link between MDM1 and the metabolic turnover of sphingoid bases. Our recent studies ${ }^{20,21}$ also found that MDM1 directly interacts with fatty acids via its hydrophobic N-terminal region and promotes lipid droplet formation. It is unclear for now whether MDM1 binds long chain sphingoid bases, but even if the direct interaction occurs, it is likely that the mechanisms are somewhat different because the head group of sphingoid bases is much more polar and hydrophilic than fatty acids.

Furthermore, we also demonstrated that the same principle can be used to study cholesterol transport in yeast using cell growth as a functional readout, highlighting the flexibility and compatibility of our approach for studying local lipid metabolism. As cholesterol plays essential roles in maintaining numerous cellular functions, switching the system to $S$. cerevisiae should offer more freedom to modulate its levels and use provide sterols, including unnatural ones.

In metabolic flux studies, our method should be able to scan multiple lipid transfer protein candidates without the requirement of detailed information on protein structures. Arguably, our design relies on biochemical conversion and hence enzymatic activities, but this requirement should be matched without difficulty thanks to the abundance of numerous lipid metabolic enzymes (Supplementary Figure 4). In future applications, we can use lipid molecules bearing a clickable motif and a diazirine which ensures crosslinking to proteins in close proximity after exposing to an orthogonal UV light to photouncaging, as have been previously demonstrated ${ }^{32}$. In this case, after photo-uncaging, crosslinking followed by 'click' chemistry with a fluorophore should enable us to visualize lipid localization over time without relying on enzymatic activities, though it will lack the information of lipid species.

In conclusion, we presented here a novel technique of delivering lipid precursors specifically to the vacuole in $S$. cerevisiae, which enabled us to visualize and track lipid recycling from the vacuole. It has also provided a novel method to bypass the sterol exclusion barrier of aerobically grown yeast. Together with mass spectrometry (MS)-based lipidomics, we have found that the MDM1 protein plays an active role in mediating sphingolipid metabolism. Collectively, our approach provides a new framework of analyzing lipid transporters without prior structural information or in vitro reconstitution.

\section{ASSOCIATED CONTENT}

\section{Supporting Information}

The Supporting Information is available free of charge on the ACS Publications website.

Supplementary figures, Materials and Methods, synthetic procedures and chemical characterizations (PDF).

\section{AUTHOR INFORMATION}

\section{Corresponding Author}

Howard Riezman - Department of Biochemistry, and National Centre of Competence in Research (NCCR) in Chemical Biology, University of Geneva, Geneva, Switzerland.

Email: howard.riezman@unige.ch

\section{Authors}

Vladimir Girik - Department of Biochemistry, University of Geneva, Geneva, Switzerland.

Suihan Feng - Department of Biochemistry, and National Centre of Competence in Research (NCCR) in Chemical Biology, University of Geneva, Geneva, Switzerland.

W. Mike Henne - Department of Cell Biology, UT Southwestern Medical Center, Dallas, TX, USA.

Hanaa Hariri - Department of Cell Biology, UT Southwestern Medical Center, Dallas, TX, USA. Current address: Department of Biological Sciences, Wayne State University, Detroit, MI, USA

\section{Author Contributions}

$\ddagger V$.G. and S.F. contributed equally in this work. S.F., V.G. and H.R. conceived the idea and designed the experiments. S.F. synthesized caged compounds. H.H. and W.M.H. created the plasmids. V.G. and S.F. performed experiments and analyzed results. W.M.H and H.R. supervised the study. S.F. and H.R. wrote the manuscript with the help of all other authors.

\section{Notes}

The authors declare no competing interests.

\section{Funding Sources}

\section{ACKNOWLEDGMENT}

This work was supported by funds from the Welch Foundation (I1873), the NIH NIGMS (GM119768), the NIDDK (R01DK126887), the Ara Parseghian Medical Research Fund, and the UT Southwestern Endowed Scholars Program (W.M.H.) and by the NCCR Chemical Biology and Swiss National Science Foundation (51NF40-185898 and 310030_184949) and the Canton of Geneva (H.R.).

\section{REFERENCES}

1. Harayama, T.; Riezman, H., Understanding the diversity of membrane lipid composition. Nat Rev Mol Cell Biol 2018, 19 (5), 281-296. 2. Wong, L. H.; Gatta, A. T.; Levine, T. P., Lipid transfer proteins: the lipid commute via shuttles, bridges and tubes. Nat Rev Mol Cell Biol 2019, 20 (2), 85-101.

3. da Silveira Dos Santos, A. X.; Riezman, I.; Aguilera-Romero, M. A.; David, F.; Piccolis, M.; Loewith, R.; Schaad, O.; Riezman, H., Systematic lipidomic analysis of yeast protein kinase and phosphatase mutants reveals novel insights into regulation of lipid homeostasis. Mol Biol Cell 2014, 25 (20), 3234-46.

4. Guan, X. L.; Souza, C. M.; Pichler, H.; Dewhurst, G.; Schaad, O.; Kajiwara, K.; Wakabayashi, H.; Ivanova, T.; Castillon, G. A.; Piccolis, M.; Abe, F.; Loewith, R.; Funato, K.; Wenk, M. R.; Riezman, H., Functional interactions between sphingolipids and sterols in biological membranes regulating cell physiology. Mol Biol Cell 2009, 20 (7), 2083-95.

5. Ejsing, C. S.; Sampaio, J. L.; Surendranath, V.; Duchoslav, E.; Ekroos, K.; Klemm, R. W.; Simons, K.; Shevchenko, A., Global analysis of the yeast lipidome by quantitative shotgun mass spectrometry. Proc Natl Acad Sci U S A 2009, 106 (7), 2136-41.

6. Feng, S.; Harayama, T.; Chang, D.; Hannich, J. T.; Winssinger, N.; Riezman, H., Lysosome-targeted photoactivation reveals local sphingosine metabolism signatures. Chem Sci 2019, 10 (8), 2253-2258.

7. Feng, S.; Harayama, T.; Montessuit, S.; David, F. P.; Winssinger, N.; Martinou, J. C.; Riezman, H., Mitochondria-specific photoactivation to monitor local sphingosine metabolism and function. Elife 2018, 7.

8. Gong, X.; Qian, H.; Zhou, X.; Wu, J.; Wan, T.; Cao, P.; Huang, W.; Zhao, X.; Wang, X.; Wang, P.; Shi, Y.; Gao, G. F.; Zhou, Q.; Yan, N., Structural Insights into the Niemann-Pick C1 (NPC1)- 
bioRxiv preprint doi: https://doi.org/10.1101/2021.05.04.442581; this version posted May 4, 2021. The copyright holder for this preprint (which was not certified by peer review) is the author/funder, who has granted bioRxiv a license to display the preprint in perpetuity. It is made available under aCC-BY-NC-ND 4.0 International license.

Mediated Cholesterol Transfer and Ebola Infection. Cell 2016, 165 (6), 1467-1478.

9. Zhang, S.; Ren, J.; Li, H.; Zhang, Q.; Armstrong, J. S.; Munn, A. L.; Yang, H., Ncr1p, the yeast ortholog of mammalian Niemann Pick C1 protein, is dispensable for endocytic transport. Traffic 2004, 5 (12), 1017-30.

10. Murphy, M. P., Targeting lipophilic cations to mitochondria. Biochim Biophys Acta 2008, 1777 (7-8), 1028-31.

11. Haak, D.; Gable, K.; Beeler, T.; Dunn, T., Hydroxylation of Saccharomyces cerevisiae ceramides requires Sur2p and Scs7p. J Biol Chem 1997, 272 (47), 29704-10.

12. Narita, T.; Naganuma, T.; Sase, Y.; Kihara, A., Long-chain bases of sphingolipids are transported into cells via the acyl-CoA synthetases. Sci Rep 2016, 6, 25469.

13. Benedetti, H.; Raths, S.; Crausaz, F.; Riezman, H., The END3 gene encodes a protein that is required for the internalization step of endocytosis and for actin cytoskeleton organization in yeast. Mol Biol Cell 1994, 5 (9), 1023-37.

14. Li, S. C.; Kane, P. M., The yeast lysosome-like vacuole: endpoint and crossroads. Biochim Biophys Acta 2009, 1793 (4), 650-63.

15. Platt, F. M., Sphingolipid lysosomal storage disorders. Nature 2014, 510 (7503), 68-75.

16. Breslow, D. K., Sphingolipid homeostasis in the endoplasmic reticulum and beyond. Cold Spring Harb Perspect Biol 2013, 5 (4), a013326.

17. Funato, K.; Lombardi, R.; Vallee, B.; Riezman, H., Lcb4p is a key regulator of ceramide synthesis from exogenous long chain sphingoid base in Saccharomyces cerevisiae. J Biol Chem 2003, 278 (9), 732534.

18. Murley, A.; Sarsam, R. D.; Toulmay, A.; Yamada, J.; Prinz, W. A.; Nunnari, J., Ltc1 is an ER-localized sterol transporter and a component of ER-mitochondria and ER-vacuole contacts. Journal of Cell Biology 2015, 209 (4), 539-548.

19. Prinz, W. A.; Toulmay, A.; Balla, T., The functional universe of membrane contact sites. Nat Rev Mol Cell Biol 2020, 21 (1), 7-24.

20. Hariri, H.; Rogers, S.; Ugrankar, R.; Liu, Y. L.; Feathers, J. R.; Henne, W. M., Lipid droplet biogenesis is spatially coordinated at ER-vacuole contacts under nutritional stress. EMBO Rep 2018, 19 (1), 57-72.

21. Hariri, H.; Speer, N.; Bowerman, J.; Rogers, S.; Fu, G.; Reetz, E.; Datta, S.; Feathers, J. R.; Ugrankar, R.; Nicastro, D.; Henne, W. M., Mdm1 maintains endoplasmic reticulum homeostasis by spatially regulating lipid droplet biogenesis. J Cell Biol 2019, 218 (4), 13191334.
22. Bryant, D.; Liu, Y.; Datta, S.; Hariri, H.; Seda, M.; Anderson, G.; Peskett, E.; Demetriou, C.; Sousa, S.; Jenkins, D.; Clayton, P.; BitnerGlindzicz, M.; Moore, G. E.; Henne, W. M.; Stanier, P., SNX14 mutations affect endoplasmic reticulum-associated neutral lipid metabolism in autosomal recessive spinocerebellar ataxia 20. Hum Mol Genet 2018, 27 (11), 1927-1940.

23. Ugrankar, R.; Bowerman, J.; Hariri, H.; Chandra, M.; Chen, K.; Bossanyi, M. F.; Datta, S.; Rogers, S.; Eckert, K. M.; Vale, G.; Victoria, A.; Fresquez, J.; McDonald, J. G.; Jean, S.; Collins, B. M.; Henne, W. M., Drosophila Snazarus Regulates a Lipid Droplet Population at Plasma Membrane-Droplet Contacts in Adipocytes. Dev Cell 2019, 50 (5), 557-572 e5.

24. Datta, S.; Liu, Y.; Hariri, H.; Bowerman, J.; Henne, W. M., Cerebellar ataxia disease-associated Snx14 promotes lipid droplet growth at ER-droplet contacts. Journal of Cell Biology 2019, 218 (4), 13351351.

25. Henne, W. M.; Zhu, L.; Balogi, Z.; Stefan, C.; Pleiss, J. A.; Emr, S. D., Mdm1/Snx13 is a novel ER-endolysosomal interorganelle tethering protein. J Cell Biol 2015, 210 (4), 541-51.

26. Pan, X.; Roberts, P.; Chen, Y.; Kvam, E.; Shulga, N.; Huang, K.; Lemmon, S.; Goldfarb, D. S., Nucleus-vacuole junctions in Saccharomyces cerevisiae are formed through the direct interaction of Vac8p with Nvj1p. Mol Biol Cell 2000, 11 (7), 2445-57.

27. Goldstein, J. L.; Brown, M. S., A century of cholesterol and coronaries: from plaques to genes to statins. Cell 2015, 161 (1), 161-172.

28. Souza, C. M.; Schwabe, T. M.; Pichler, H.; Ploier, B.; Leitner, E.; Guan, X. L.; Wenk, M. R.; Riezman, I.; Riezman, H., A stable yeast strain efficiently producing cholesterol instead of ergosterol is functional for tryptophan uptake, but not weak organic acid resistance. Metab Eng 2011, 13 (5), 555-69.

29. Keesler, G. A.; Casey, W. M.; Parks, L. W., Stimulation by heme of steryl ester synthase and aerobic sterol exclusion in the yeast Saccharomyces cerevisiae. Arch Biochem Biophys 1992, 296 (2), 474-81. 30. Marcireau, C.; Guilloton, M.; Karst, F., In vivo effects of fenpropimorph on the yeast Saccharomyces cerevisiae and determination of the molecular basis of the antifungal property. Antimicrob Agents Chemother 1990, 34 (6), 989-93.

31. Vallée, B. and Riezman, H. Lip1p: a novel subunit of acyl-CoA ceramide synthase. EMBO J., 2005, 24(4), 730-741.

32. Hoglinger, D.; Nadler, A.; Haberkant, P.; Kirkpatrick, J.; Schifferer, M.; Stein, F.; Hauke, S.; Porter, F. D.; Schultz, C., Trifunctional lipid probes for comprehensive studies of single lipid species in living cells. Proc. Natl. Acad. Sci., 2017, 114 (7), 1566-1571. 
bioRxiv preprint doi: https://doi.org/10.1101/2021.05.04.442581; this version posted May 4, 2021. The copyright holder for this preprint (which was not certified by peer review) is the author/funder, who has granted bioRxiv a license to display the preprint in perpetuity. It is made available under aCC-BY-NC-ND 4.0 International license.

\section{Graphic Abstract}
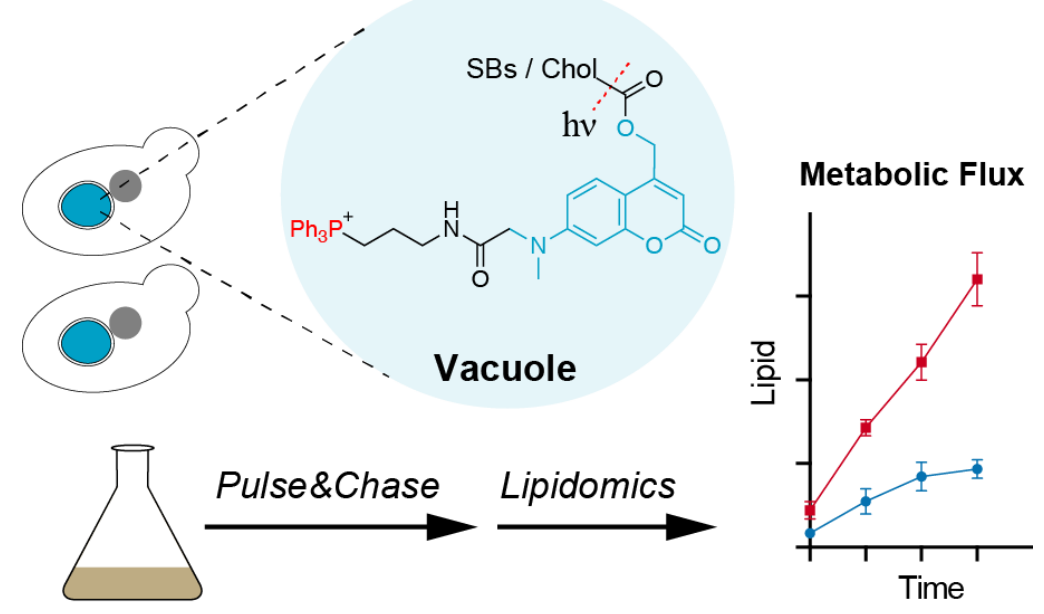